VOL. $20(1979), 35-55$.

\title{
An algebraic theory of hypergroups
}

\section{J.R. McMullen}

\begin{abstract}
The category of groups forms a full subcategory of the category of hypergroups. This larger category also contains other group theoretic objects, such as the conjugacy class hypergroup and character hypergroup of a finite group.

Definitions of hypergroups and hypergroup morphisms are given and related to double algebras (which are simultaneously algebras and cogebras, but not Hopf algebras in general). Quotient and orbit hypergroups are defined. Coefficients are allowed in a more or less arbitrary field.

These concepts provide a new language in which groups and their character tables can be fruitfully discussed.
\end{abstract}

\section{Introduction}

It is classical that the characters of a compact abelian group form a discrete abelian group. In recent years, harmonic analysts have endowed the dual of even a compact nonabelian group with an algebraic structure in order to exploit analogies with the abelian case. Thus the hypergroup has been born, and with its sister the locally compact topological hypergroup toils daily in the harmonic-analytic vineyard.

Despite their algebraic nature, however, an appropriate algebraic treatment of discrete hypergroups has not appeared until now, although the literature is already of significant size, as perusal of the bibliography of [6] shows (see especially [8], an analytic treatment, and [7] for an algebraic notion of hypergroup with weaker structure).

In presenting what we feel is such a treatment, there are two

Received 8 November 1978. 
additional motives. In [1], Braver introduced the idea of studying character tables in abstracto, by considering them as square arrays of complex numbers satisfying certain postulates. It was shown in [2] that such character tables, though not always having an underlying group, always have an underlying hypergroup. Hence the hypergroup suggests itself as a tool for group theorists: this is our first motive. Our second motive is to illustrate the satisfying way in which hypergroup theory extends group theory.

Accordingly, we give herein definitions of hypergroups and their morphisms, and their relation to double algebras ( $\$ 1)$. Quotient hypergroups ( $\$ 2)$, and finally orbit hypergroups (\$3) are studied. This selection of topics is made with algebraic readers in mind. Much of the treatment allows coefficients in a more or less general ring.

A duality theorem for finite abelian hypergroups over a splitting field is presented in a companion paper [3], which logically depends only on $\$ 1$ of the present paper.

\section{Hypergroups and hypergroup algebras}

1.0 .

Let $R$ be a fixed commutative ring with identity $I$. Let $C$ be a subset of $R$. A hypergroup $(H, n, d, 1,-)$ with coefficients in $C$ is a set $H$ together with three maps $n: H^{3} \rightarrow C, d: H \rightarrow C \backslash\{0\}$, - : $H \rightarrow H$, called multiplicity, degree, and conjugation, respectively, together with a distinguished element $l \in H$, satisfying the following axioms :

(HI) (Associativity) for $a, b \in H, n(a, b, x) \neq 0$ for only finitely many $x \in H$, and

$$
\sum_{x \in H} n(a, b, x) n(x, c, f)=\sum_{y \in H} n(b, c, y) n(a, y, f)
$$

(H2) (Identity) for $a, b \in H, n(I, a, b)=n(a, 1, b)=\delta_{a b}$;

(H3) (Reversibility) for $a, b \in H, n(a, \bar{b}, 1) \neq 0$ if and only if $a=b$; and for all $a \in H$, $n(a, \bar{a}, 1)=n(\bar{a}, a, 1)$; 
(H4) (Conjugation) $n(\bar{a}, \bar{b}, \bar{c})=n(b, a, c) ; d(\bar{a})=d(a)$;

(H5) (Degree) $d(a) d(b)=\sum_{x \in H} n(a, b, x) d(x)$;

(H6) (Division) $n(a, \bar{a}, 1)$ divides $d(a)^{2}$ in $R$, for each $a \in H$, and the quotient belongs to $C$.

Elementary examples of hypergroups are the character hypergroup $\hat{G}$ of a compact group $G$, and conjugacy class hypergroup $\bar{G}$ of a group $G$ whose conjugacy classes are finite. These are abelian hypergroups in that they satisfy the identity

$$
n(a, b, c)=n(b, a, c),
$$

and their coefficients are nonnegative integers. Every finite group is also a hypergroup.

It follows from ( $\mathrm{HI}),(\mathrm{H} 3)$, and (H4) that the identity

$$
n(a, b, c) r(c)=n(\bar{a}, c, b)_{r}(b)=n(c, \bar{b}, a) r(a)
$$

holds, where $r(a)=n(a, \bar{a}, 1)=n(\bar{a}, a, 1)$. This explains the word "reversible". Notice that if $G$ is a finite group, then for $\hat{G}, r \equiv I$, while for $\bar{G}, r(x)=d(x)$ for $x \in \bar{G}$. Such hypergroups are called standard and classlike, respectively.

\subsection{REMARKS ON THE AXIOMS}

(i) If $d(a)=1$ for all $a$, and if the coefficients are real and nonnegative, then our notion of a hypergroup coincides with that of a discrete hypergroup in Spector [4], [5]. We call a hypergroup grouplike if $d(a)=1$ for all $a \in H$. All hypergroups are equivalent in a certain sense with a grouplike hypergroup, as demonstrated in Proposition 1 below.

(ii) If $H$ is finite and has positive real coefficients then $d$ is unique. This follows from the orthogonality relations [3, Section 8$]$.

(iii) Every abelian hypergroup has an underlying "canonical hypergroup" structure (see [7]) when we define the hyperproduct by

$$
a \times b=\{x \in H \mid n(a, b, x) \neq 0\} .
$$

However, there exists a canonical hypergroup with no corresponding hypergroup structure, if we require the coefficients to be integers.

(iv) It follows from the axioms that $\overline{\bar{a}}=a$ for $a \in H$ and that $\bar{a}$ 
is unique.

\subsection{NORM OF A HYPERGROUP}

This is defined for a finite hypergroup by

$$
\|H\|=\sum_{a \in H} d(a)^{2} r(a)^{-1}
$$

which by $(\mathrm{H} 6)$ is an element of the coefficient ring. It is analogous to the order of a group, and coincides with this concept if $H$ is a group.

\subsection{GROUP OF SCALARS}

Let $H$ be a hypergroup with positive integral coefficients, and let $a \in H$. Then adapting [2, Lemma 2.4] we see that the conditions

$$
\begin{aligned}
& \text { (i) } d(a)=1, \\
& \text { (ii) } n(a, \bar{a}, b)=\delta_{1 b} \text { for } b \in H \text {, and } \\
& \text { (iii) for each } b \in H \text { there exists a unique } \varepsilon \in H \text { such that } \\
& n(a, b, x)=\delta_{c x} \text { for all } x \in H,
\end{aligned}
$$

are all equivalent (note that by $(\mathrm{H} 6), d(a)=1$ implies $r(a)=1$ ). The set of such elements forms a group if we put $a \cdot b=c$ when (iii) holds. This group is called the group of scalars of $H$.

Note that the three conditions certainly are not equivalent if the ceofficients are not assumed to be integral and positive.

\subsection{HYPERGROUP ALGEBRAS}

Every group may be regarded as a standard classlike hypergroup with positive integral coefficients (and conversely, by 1.3). Just as a group gives rise to its group Hopf algebra, a hypergroup gives rise to a "double algebra", which we now define. It is of great theoretical importance and implies the duality theorem of [3].

A double algebra over a field $k$ is a $k$ module $A$ with the following structure (see [9] for terminology on cogebras):
(A工) $A$ is a $k$-algebra with unit $u: k \rightarrow A$;
(A2) $A$ is a $k$-cogebra with counit $d: A \rightarrow k$;
(A3) the grouplikes in the cogebra $A$ span $A$ (hence are a 
basis);

(A4) $u$ is a cogebra map;

(A5) $d$ is an algebra map;

(A6) there is a mapping $S: A \rightarrow A$ which is a cogebra map, and an algebra map of $A$ into its opposite algebra $A^{0}$ (it follows that $S$ maps grouplikes to grouplikes and $S(1)=1$ );

(A7) let $\theta_{1}: A \rightarrow k$ be the unique $k$-linear map such that $\theta_{1}(x)$ is the coefficient of 1 when $x$ is written in terms of grouplikes. Then for grouplikes $a, b \in A$, $\theta_{1}(b S(a))=\theta_{1}(a S(b)) \neq 0$ if and only if $a=b$ (it follows that $S^{2}=I_{A}$ and that $S$ is unique).

PROPOSITION 1. Let $H$ be a hypergroup with coefficients in a field $k$. Let $k H$ be the free k-module with basis $H$. Define multiplication on $k H$ by $(a, b) \rightarrow \sum_{c \in H} n(a, b, c) c$, let $u(1)=1 \in H$, and let $\Delta, d, S$ be the unique $k$-linear extensions of the maps

$$
\begin{aligned}
& \Delta: a \rightarrow d(a)^{-1} a \otimes a \quad(a \in H), \\
& d: a \rightarrow d(a) \quad(a \in H), \\
& S: a \rightarrow \bar{a}(a \in H) .
\end{aligned}
$$

Then $k H$ is a double algebra, and $\left\{d(a)^{-1} a \mid a \in H\right\}$ is its set of groupiikes.

Conversely, let $A$ be a double algebra, and let $H_{0}(A)$ be the set of grouplikes in $A$. Then there is a unique hypergroup structure on $H_{0}(A)$ for which $A$ is isomorphic to $k\left(H_{0}(A)\right)$ both as algebra and cogebra.

The proof is a trivial consequence of the axioms.

\subsection{MORPHISMS}

A morphism $A \stackrel{\Phi}{\longrightarrow} B$ between two double algebras over the same field 
$k$ is a $k$-linear map which is an algebra map, a cogebra map, and such that $S \circ \phi=\phi \circ S$. A morphism necessarily preserves grouplikes. A morphism $H \stackrel{\Phi}{\rightarrow} J$ between two hypergroups $H$ and $J$ with coefficients in $k$ is a morphism $k H \stackrel{\phi}{\longrightarrow} k J$ of the corresponding double algebras. By isomorphism we mean a morphism possessing an inverse morphism.

If $R \leq k$ is a subring of $k$ then $\phi$ is a $R$-morphiam if $\phi(R H) \subseteq R J$ (that is if $\phi(H) \subseteq R J$ ). A weak $R$-morphism is an algebra map $\phi: R H \rightarrow R J$ preserving the counit and $S$.

Note that if $\phi: H \rightarrow J$ is an $R$-morphism of hypergroups, then $\phi(a)$ is an $R$-multiple of an element of $J$, for each $a \in H$.

PROPOSITION 2. (i) Let $A=k H$ be a double algebra. Let $H_{2}(A)$ be the set of classlike elements of $A$, that is, those of the form $d(a)_{r}(a)^{-1} a \quad(a \in H)$. Then $H_{2}(A)$ is independent of the original hypergroup $H$, and under its induced hypergroup structure $H_{2}(A)$ is a classlike hypergroup.

(ii) Let $A=k H$ be a double algebra, where $H$ has positive real coefficients. Let $H_{I}(A)$ denote the set of standard elements of $A$, namely those of the form $r(a)^{\frac{1}{2}} \alpha(a \in H)$, where $r(a)^{\frac{3}{2}}$ denotes the positive square root. Then $H_{1}(A)$ is independent of the original hypergroup $H$, and under its induced hypergroup structure $H_{1}(A)$ is a standard hypergroup.

Proof. It is easy to see that $H_{1}(A)$ and $H_{2}(A)$ may be defined in terms of the grouplike hypergroup $H_{0}(A)$. The rest follows.

COROLLARY. If $H$ is a hypergroup with coefficients in a field $k$, then there is a classlike hypergroup with coefficients in $k$ which is $k$-isomorphic with $H$. If $k$ contains the field $\mathbf{R}$ and $H$ has positive real coefficients, then there is also a standard hypergroup with these properties.

We stress here that a morphism $\phi: H \rightarrow J$ of hypergroups - even an isomorphism - is not necessarily a set-theoretic mapping of $B$ into $J$, 
save as indicated in the following proposition.

PROPOSITION 3. Let $\phi: A \rightarrow B$ be a morphism of double algebras. Then $\phi$ maps grouplikes to grouplikes, and classlikes to classlikes. If $x \in A$ is standard, then there exists a standard $y \in B$ such that $\phi(x)=d(x) d(y)^{-1}(y)$. If $\phi$ is injective, and $x \in A$ is standard, then $\phi(x)$ is standard.

\subsection{EXAMPLES OF MORPHISMS}

(i) $\{1\}$ is a hypergroup in a unique way and so $k \cong k\{1\}$ is a double algebra (in fact, a Hopf algebra). If $H$ is any hypergroup then the unit and counit $d: k H \rightarrow k$ and $u: k \rightarrow k H$ are morphisms.

(ii) Let $\phi: G_{1} \rightarrow G_{2}$ be a continuous group homomorphism of compact groups. For $i=1,2, \hat{G}_{i}$ can be identified with an algebra of functions on $G_{i}$. Put $\hat{\phi}(f)=f \circ \phi$. Then $\hat{\phi}$ is a weak $\mathbb{Z}^{+}$-morphism from $\hat{G}_{2}$ to $\hat{G}_{1}$, and is a $\mathbb{Z}^{+}$-morphism precisely when $\phi$ is surjective.

This exhibits $\wedge$ as a functor from the opposite category of the category of compact groups and continuous group homomorphisms to the category of standard abelian hypergroups with positive integral coefficients and weak $\mathbb{Z}^{+}$-morphisms.

\subsection{SUBHYPERGROUPS}

Let $H$ be a hypergroup. A subset $J$ of $H$ is a subhypergroup if $a, b \in J$ and $c \notin J$ imply $n(a, b, c)=0$ and $S(J) \subseteq J$. The subset $J$ then inherits a hypergroup structure from $H$ in the obvious way, and $k J$ is a subalgebra of $k H$ which is simultaneously a subcogebra.

Conversely, if $B \subseteq k H$ is a subalgebra and subcogebra of $k H$, then it is easily seen that if $x \in B$ and $x=\sum_{a \in H} \alpha(a) a$ then for all $a \in H$ either $\alpha(a)=0$ or $a \in B$. Thus $B$ is the linear span of some subset $J$ of $H$, which must be a subhypergroup since $B$ is a subalgebra.

If $G$ is a group, then $J \subseteq G$ is a subhypergroup if and only if it is a subgroup.

It is certainly clear that if $\phi: H+K$ is a morphism, then there is a subhypergroup $J$ of $K$ with inclusion morphism $i$, and a morphism 
$\psi: H \rightarrow J$ such that

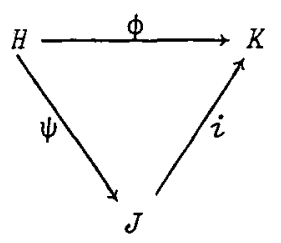

commutes, and $\psi(k H)=k J$.

1.8. DOUBLE ALGEBRAS WHICH ARE BIGEBRAS

The following is now easy to prove.

THEOREM 1. Let $H$ be a hypergroup with positive integral coefficients. Let $A=\mathbb{Q} H$ be the rational double algebra of $H$ isee Proposition 1). Then the following are equivalent:

(i) $A$ is a Hopf algebra and $S$ is its antipode;

(ii) $A$ is a Hopf algebra;

(iii) $A$ is a bigebra;

(iv) $\Delta$ is an algebra map;

(v) $M$ is a cogebra map;

(vi) $S * I=I * S=$ ud where * denotes convolution in $\operatorname{hom}_{\varphi}(A, A)$;

(vii) elements of $H$ are invertible in $A$;

(viii) $H_{0}(A)$ is a group.

\section{Quotient hypergroups}

\subsection{QUOTIENTS}

Let $A$ be a double algebra. Then a double ideal in $A$ is a $k$ submodule $V$ of $A$ which is

(1) an ideal,

(2) a coideal (see $[9, p .18 \mathrm{ff}]$ ), and

(3) invariant under $S$.

The kernel of the counit $d$ is the largest coideal, and since it is 
also an ideal invariant under $S$ it is the largest double ideal. We plan to establish a correspondence between double ideals in $k H$ of a certain type and completely normal subhypergroups of $H$.

PROPOSITION 4. Let $k H$ be a double algebra, let $V \subseteq k H$ be a double ideal, and let $\pi: k H \rightarrow k H / V=E$ be the natural k-linear map.

(a) There is a unique structure of algebra and cogebra on $E$ such that $\pi$ is a morphism of both algebras and cogebras, and a unique mapping $S: E \rightarrow E$ with $S \circ \pi=\pi \circ S$. With these stmuctures, $E$ satisfies all axioms for a double algeira except (A7).

(b) If $f: k H \rightarrow D$ is any map into a k-module $D$ which has the above three structures and if $f$ respects these stmuctures then ker $f$ is a double ideal.

(c) If $V \subseteq$ ker $f$ then there is a unique k-module morphism $f^{*}$ such that the diagram

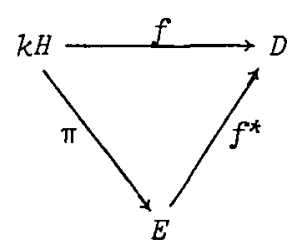

commutes.

The proof is trivial.

PROPOSITION 5. Let $H$ be a hypergroup with positive real coefficients contained in the field $k$. Let $V$ be a double ideal in the double algebra $k H$. Let $\pi: k H \rightarrow k H / V=E$ be the quotient map. Let $J$ be the set

$$
J=\{a \in H \mid \pi(a)=d(a) .1\} \text {. }
$$

Thus $J$ is a subhypergroup of $H$. Also, $E$ is a double algebra, that is satisfies axiom (A7), if and only if $J$ satisfies the following condition:

(a) for alz $a, b \in H$,

$$
\sum_{z \in J} n(a, b, z) d(z)=\sum_{z \in J} n(b, a, z) d(z) .
$$

If $J$ is finite, then (a) is equivalent to: 
(b) for all $a \in H$,

$$
\xi_{J} a=a \xi_{J}
$$

where $\xi_{J}=\sum_{z \in J} d(z) r(z)^{-1} z \in k J$.

Proof. Obviously $\vec{J} \subseteq J$. Suppose $n(a, b, c) \neq 0$ with $a, b \in J$. Then we have $\pi(a b)=\pi(a) \pi(b)$, which is a multiple of $1 \in E$, while $\pi(a b)=\sum_{c^{\prime} \in H} n\left(a, b, c^{\prime}\right) \pi\left(c^{\prime}\right)$, so that $n(a, b, c) \neq 0$ entails that $\pi(c)$ is a multiple of 1 . Thus $J$ is a subhypergroup of $H$.

It is a simple exercise to show that if $J$ is finite then (a) and (b) are equivalent.

Now, as the grouplikes in $E$ are the elements $d(x)^{-1} \pi(x)$, where $x$ is a grouplike in $k H,($ A 7 ) for $E$ is equivalent to axiom (H3) for elements $\pi(a)$, namely, to the assertions that for $a, b \in H$, $n(\pi(a), \pi(b), 1)=n(\pi(b), \pi(a), 1)$ and that this expression takes the value 0 if and only if $\pi(a) \neq \overline{\pi(b)}$. The first assertion is equivalent to $\left(^{*}\right)$. The proof will be complete if we prove that the second assertion always holds.

Now observe that $n(\pi(a), \pi(b), 1)=\sum_{z \in J} n(a, b, z) d(z)=r(b) \alpha$, where $a$ is the coefficient of $b$ in $\bar{a} \xi_{J}$. Also, $\pi\left(\xi_{J}\right)=\|J\| . I$, so that $\pi\left(a \xi_{j}\right)=\|J\| \overline{\pi(a)}$. If $n(\pi(a), \pi(b), 1) \neq 0$ then $\alpha \neq 0$, and hence by the linear independence of grouplikes, $\pi(b)=\overline{\pi(a)}$. Conversely $\begin{aligned} n(\pi(a), \overline{\pi(a)}, 1)=n(\pi(a), \pi(\bar{a}), 1) & \\ & =\sum_{z \in J} n(a, \bar{a}, z) d(z) \geq n(a, \bar{a}, 1)>0 .\end{aligned}$

We shall call a subhypergroup prenormal, if it satisfies condition (a) of Proposition 5. If $V$ is a double ideal in $k H$, the subhypergroup defined in Proposition 5 is said to be associated with $V$. The ideal $V$ is generated as a $k$-module by the elements $d\left(a^{\prime}\right) a-d(a) a^{\prime}$ where $a, a^{\prime} \in H$ and $d(a) \pi\left(a^{\prime}\right)=d\left(a^{\prime}\right) \pi(a)$.

PROPOSITION 6. Let $k, H, V, \pi$, and $J$ be as in Proposition 5, and 
suppose that $J$ is prenormal. Then the following are equivalent for $a, b \in H:$

(i) $d(a) \pi(b)=d(b) \pi(a)$;

(ii) $n(\pi(a), \overline{\pi(b)}, 1) \neq 0$;

(iii) there exists $z \in J$ such that $n(b, z, a) \neq 0$;

(iv) there exists $z \in J$ such that $n(a, \bar{b}, z) \neq 0$;

and if $J$ is finite, these are equivalent to

(v) the coefficient of $b$ in $a \xi_{J}$ is nonzero;

(vi) the coefficient of $a$ in $b \xi_{J}$ is nonzero.

The conditions ( $i i i)$ and (iv) are plainly equivalent whatever the prenormal subhypergroup $J$ of $H$. Let us write $a \sim b$ when these conditions hold. It is elementary to show that $\sim$ is an equivalence relation. A $\sim$ class we call a coset modulo $J$.

Proposition 5 can be stated in the following form.

PROPOSITION $5^{\prime}$. If $H$ is a hypergroup with positive real coefficients in a field $k$ and if $k H \stackrel{\alpha}{\longrightarrow} k L$ is a hypergroup morphism surjective as a $k$-linear map (that is, as a cogebra map) then the largest subcogebra $k J \stackrel{\iota}{\longrightarrow} k H$ for which the diagram

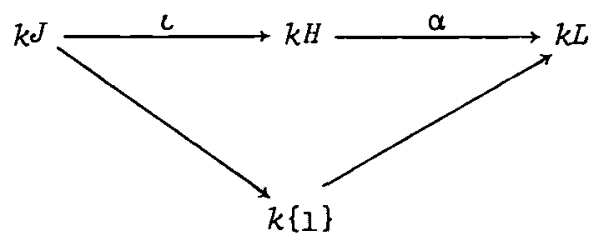

commutes is actually a subalgebra as well, so that $J$ is a (prenormal) subhypergroup and further, any commtative diagram

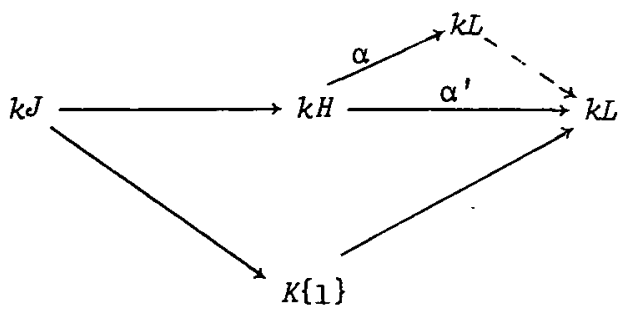


where $\alpha$ and $\alpha^{\prime}$ are morphisms can be completed commutatively as shown. In particular, all morphisms have kernels.

The dual problem is the following. If $k J \stackrel{l}{\longrightarrow} k H$ is a prenormal subhypergroup, what conditions on $J$ ensure that it is a kernel, that is, that there is a morphism with domain $k H$ such that $(\dagger)$ commutes? We call the prenormal subhypergroup $J$ normal if this is the case. The equivalence of this to the following conditions is routine to check $((3)-(6)$ assuming $J$ is finite, (7), (8) assuming $h$ abelian):

(1) for all $a, b, c \in H$, the element

$$
\sum_{c^{\prime} \sim c} n\left(a, b, c^{\prime}\right) d\left(c^{\prime}\right) d(a)^{-1} d(b)^{-1}
$$

of $k$ depends only on the cosets modulo $J$ to which $a$ and $b$ belong;

(2) the $k$-linear span $V$ of the set $\left\{d(a)^{-1} a-d(b)^{-1} b \mid a \sim b\right\}$ is an ideal in $k H$;

(3) for all $a \in H, d(a)^{-1} a \xi_{J}=d\left(\xi_{J}\right) d\left(\xi_{a J}\right)^{-1} \xi_{a J}$, where $\xi_{a J}=\sum_{a^{\prime} \sim a} d\left(a^{\prime}\right) r\left(a^{\prime}\right)^{-1} a^{\prime}$

(4) $V$ is the annihilator of $\xi_{J}$;

(5) $a \sim b \Leftrightarrow d(a)^{-1} a \xi_{J}=d(b)^{-1} b \xi_{J}$ (note that as $d\left(\xi_{J}\right)^{-1} \xi_{J}$ is idempotent, $a \mapsto d\left(\xi_{J}^{-1}\right) a \xi_{J}$ is always a ring homomorphism ;

(6) the set $\left\{d(a)^{-1} a \xi_{J} \mid a \in H\right\}$ is $k$-linearly independent;

(7) $J=A^{\perp}=\{a \in H \mid \forall \rho \in A, \rho(a)=d(a)\}$ for some set $A$ of algebra maps $k H \rightarrow k$;

(8) $J=J^{1 \perp}$.

The following is an extension of the well-known property of abelian groups.

PROPOSITION 7. Let $H$ be an abelian hypergroup with positive real coefficients in a splitting field $k$ whose grouplike dual $H_{0}^{*}$ also has 
positive coefficients (refer to [3, paragraph 14, proposition]). Then every subhypergroup of $H$ is normal.

Proof. It is proved in [3] that the category $A$ of abelian hypergroups (equivalently, of commutative double algebras) with real coefficients and morphisms is self dual, that is to say that $D: H \rightarrow H_{0}^{*}$ (equivalently, $k H \rightarrow(k H)^{*}$ ) and defined in the obvious way on morphisms is a functor from this category to its opposite category, and the isomorphisms ${ }_{H}: H \rightarrow H^{* *}$ constitute an equivalence of the identity functor on $A$ with $D^{2}$.

The hypergroups with positive real coefficients contained in a fixed splitting field $k \subseteq \mathbb{C}$, and whose duals have positive real coefficients form a full subcategory $A_{k}^{+}$of $A$, and $D A_{k}^{+}=\left(A_{k}^{+}\right)^{\circ p}$.

Let us call a morphism $H \stackrel{\alpha}{\longrightarrow} L$ of hypergroups surjective or injective according to whether it is surjective or injective on the underlying sets of grouplikes (equivalently, on the $k$ modules $k H$ and $k L$ ).

If $A$ is a semisimple commutative algebra over a splitting field $k$ and if $A_{1} \subseteq A$ is a $k$-subalgebra, then every algebra map $\rho_{1}: A_{1} \rightarrow k$ extends to an algebra map $\rho: A \rightarrow k$. Consequently, a morphism $H \stackrel{\alpha}{\longrightarrow} L$ in $A_{k}^{+}$is injective if and only if its dual $L_{0}^{*} \stackrel{\alpha^{*}}{\longrightarrow} H_{0}^{*}$ is surjective. Combining these facts with Proposition $5^{\prime}$ we obtain Proposition 7.

The hypotheses of Proposition 7 are satisfied by $\bar{G}$ and $\hat{G}$ if $G$ is a finite group. The category $A_{k}^{+}$is also closed under subhypergroups and morphic images.

The cokernel arising from a subhypergroup $J \subseteq H$ (if $H \in A_{k}^{+}$) we will denote by $H / J$, and call the quotient hypergroup of $H$ by $J$. Note that $(H / J)^{*}$ is isomorphic with the subhypergroup $J^{\perp}$ of $H^{*}$.

\subsection{COEFFICIENTS OF A QUOTIENT HYPERGROUP}

Let $H$ be a hypergroup with positive integral coefficients, and let $J$ be a normal finite subhypergroup of $H$. Plainly, each coset of $J$ is then finite. Let $V$ be the double ideal with which $J$ is associated, and 
$\pi$ the quotient map $Q H \rightarrow \mathbb{Q} H / V=E$.

$$
\begin{aligned}
& \text { For } a \in H \text {, define } a_{J}=d\left(a_{J}\right) d(a)^{-1} \pi(a) \text {, where } \\
& d\left(a_{J}\right)=\text { g.c.d. }\{d(x) \mid x \in H, x \sim a\} .
\end{aligned}
$$

Let $H / J$ be the hypergroup $\left\{a_{J} \mid a \in H\right\}$, whose double algebra is $E$. Put

$$
\left\|a_{j}\right\|=\sum_{a^{i} \sim a} d\left(a^{\prime}\right)^{2} r\left(a^{\prime}\right)^{-1}
$$

PROPOSITION 8. The following statements are true:

(i) $n\left(a_{J}, b_{J}, c_{J}\right)$

$$
=d\left(a_{J}\right) d\left(b_{J}\right) d(a)^{-1} d(b)^{-1} d\left(c_{J}\right)^{-1} \sum_{c^{i} \sim c} n\left(a, b, c^{\prime}\right) d\left(c^{\prime}\right) ;
$$

(ii) $r\left(a_{J}\right)=n\left(a_{J}, a_{J}, 1\right)=\|J\| d\left(a_{J}\right)^{2}\left\|a_{J}\right\|^{-1}$;

(iii) $\|H\|=\|J\|\|H / J\|$;

(iv) $n\left(a_{J}, b_{J}, c_{J}\right) \in \mathbb{Z}^{+}, d\left(a_{J}\right) \in \mathbb{Z}^{+}$; if $\|J\|$ is a divisor of $\left\|a_{J}\right\|$ for all $a_{J} \in H / J$, then $H / J$ has its coefficients in $\mathbb{Z}^{+}$.

Proof. Statement (i) is trivial. Multiplying it by $\frac{d(a)^{2}}{r(a)} \frac{d(b)^{2}}{r(b)}$ and summing over the cosets of $a$ and $b$ we obtain the symmetric expression

$$
\begin{aligned}
& n\left(a_{J}, b_{J}, c_{J}\right) \\
& \quad=d\left(a_{J}\right) d\left(b_{J}\right)\left\|a_{J}\right\|^{-1}\left\|b_{J}\right\|^{-1} d\left(c_{J}\right)^{-1} \sum_{a^{\prime} \sim a} \sum_{b^{\prime} \sim b} \sum_{c^{\prime} \sim c} \frac{n\left(a^{\prime}, b^{\prime}, c^{\prime}\right) d\left(c^{\prime}\right) d\left(a^{\prime}\right) d\left(b^{\prime}\right)}{r\left(a^{\prime}\right) r\left(b^{\prime}\right)}
\end{aligned}
$$

whence

$$
n\left(\dot{a}_{J}, b_{J}, c_{J}\right) \cdot d\left(c_{J}\right)^{2}\left\|c_{J}\right\|^{-1}=n\left(c_{J}, \bar{b}_{J}, a_{J}\right) d\left(a_{J}\right)^{2}\left\|a_{J}\right\|^{-1}
$$

and putting $b=\bar{a}$ and $c=1$, and using $\left\|1_{J}\right\|=\|J\|$, we obtain (ii).

$$
\text { Relation (iii) is obtained directly from ( } i i) \text {, and there remains the }
$$


proof of (iv). Now it is plain that $n\left(a_{J}, b_{J}, c_{J}\right) \geq 0$ for all $a, b, c \in H$, and that $d\left(a_{J}\right) \in\{1,2,3, \ldots\}$ by its definition.

Let $a \in H$, and let $\left\{a_{1}, \ldots, a_{p}\right\}$ be the coset of $a$. Then by the definition of $d\left(a_{J}\right)$, there are integers $k_{1}, \ldots, k_{r}$ such that

$$
d\left(a_{J}\right)=\sum_{i=1}^{r} k_{i} d\left(a_{i}\right)
$$

Therefore

$$
\pi\left(\sum_{i=1}^{r} k_{i} a_{i}\right)=\left[\sum_{i=1}^{r} k_{i} d\left(a_{i}\right)\right] d\left(a_{J}\right)^{-1} a_{J}=a_{J},
$$

showing that $a_{J}$ belongs to the ring $\pi(\mathbb{Z} H)$. It follows that $n\left(a_{J}, b_{J}, c_{J}\right)$ is an integer for all $a, b, c \in H$.

\section{3. $\|J\|$ NEED NOT DIVIDE $\|H\|$}

Consider the hypergroup

$$
H=\left\{1, a, b: d(a)=1, d(b)=5, a^{2}=1, a b=b a=b, b^{2}=5+5 a+3 b\right\rangle .
$$

It is classlike abelian with integral coefficients, with norm $\|H\|=7$. The subhypergroup $J=\{1, a\}$ has norm $\|J\|=2$. The quotient $H / J$. is given by

$$
H / J=\left\langle 1, b_{J}: d\left(b_{J}\right)=5, b_{J}^{2}=10+3 b_{J}\right\rangle \text {, }
$$

of course, $\|H / J\|=7 / 2$, so that $H / J$ does not have coefficients in $\mathbb{Z}$.

However, if $H$ is an abelian hypergroup with integral coefficients, and some form of the dual of $H$ also has integral coefficients (for example, $H_{0}^{*}, H_{1}^{*}$, or $H_{2}^{*}$ ) then every subhypergroup $J \subseteq H$ has norm dividing $\|H\|$. The reason is, of course, that $\|H / J\|=\left\|(H / J)^{*}\right\|=\left\|J^{\perp}\right\|$, where $J^{\frac{1}{}}$, the annihilator of $J$ in $H^{*}$, is a subhypergroup of $H^{*}$. By hypothesis its norm is an integer.

\section{Orbit hypergroups}

Throughout this section, hypergroups are assumed for convenience to 
have positive integral coefficients, although this is essential only in those paragraphs which deal with quotient hypergroups.

3.1 .

An automorphism of a hypergroup $H$ is an isomorphism of $H$ onto $H$ (see 1.5). The automorphisms of $H$ form a group aut $(H)$. If $H$ and $H^{\prime}$ are isomorphic, then so are aut $(H)$ and aut $\left(H^{\prime}\right)$.

3.2 .

PROPOSITION 9. Let $H^{*}$ be any dual of the abelian hypergroup $H$. Then the mapping $\phi \rightarrow\left(\phi^{*}\right)^{-1}$ is a group isomorphism of aut $(H)$ with $\operatorname{aut}\left(H^{*}\right) \quad\left(\left(\phi^{*}\right)^{-1}\right.$ has the effect of carrying the element $\rho \in H^{*}$ to the element $\rho \circ \phi^{-1}$ ).

3.3.

Let $G$ be a group. A G-hypergroup $H$ is a hypergroup upon which $G$ acts on the left by means of automorphisms. We say $H$ is trivial if the action of $G$ on $H$ is trivial. If $H$ and $H^{\prime}$ are G-hypergroups, then a G-morphism $\phi: H \rightarrow H^{\prime}$ is a morphism of hypergroups rendering the diagram

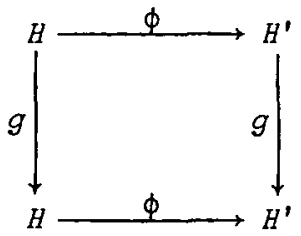

commutative for each $g \in G$. Clearly, if $H$ is a $G$-hypergroup, then $G$ acts naturally on $R H$ for any ring $R \supseteq \mathbb{Z}$ as a group of algebra and cogebra morphisms, each of which respects the operator $S$.

We may also define a weak $\left(\mathbb{Z}^{+}, G\right)$-morphism to be a weak $\mathbb{Z}^{+}$-morphism rendering (3.1) commutative.

3.4 .

In the following, \#X denotes the cardinality of the set $X$.

PROPOSITION 10. Let $H$ be a G-hypergroup, and suppose that the orbits of $H$ under $G$ are finite. Let $G \backslash H$ denote the set of orbit sums 
in $\mathbb{Z} H$ of the form

$$
[a]=\sum_{a^{\prime} \in G . a} a^{\prime} \quad(a \in H) .
$$

Then $\{[a]: a \in H\}$ is linearly independent in $\mathbb{Q} H$ and under the ring multiplication in $\mathbb{Z} H$ we have

$$
[a][b]=\sum_{[c] \in G \backslash H}\left(\sum_{a^{\prime} \in G . a} \sum_{b^{\prime} \in G . b} n\left(a^{\prime}, b^{\prime}, c\right)\right\}[c]
$$

and also

$$
d([a])=\#[a] d(a) .
$$

Then $G \backslash H$ is a hypergroup with coefficients in $\mathbb{Z}^{+}$, and

$$
r([a])=\#[a] r(a),
$$

$\|G \backslash H\|=\|H\|$ if $H$ is finite.

Proof. Relation (3.2) is obtained directly, (3.3) is obvious. Now occurs in $[a][b]$ in the decomposition (3.2) if and only if for some $g_{1}, g_{2} \in G$, we have $n\left(g_{1} a, g_{2} b, 1\right) \neq 0$; that is $g_{1} a=g_{2} \bar{b}$. This means that $[a]=[\bar{b}]$, and so $G \backslash H$ is a hypergroup. Finally, (3.4) is computed directly from the relation $r([\alpha])=n([a],[\bar{a}], 1)$.

3.5 .

We shall call $G \backslash H$ the orbit hypergroup of $H$ under $G$. Clearly, for any ring $R \supseteq \mathbb{Z}, R(G \backslash H)$ is the set of fixed points of the double algebra $R H$ under the action of $G$.

If $\phi: H \rightarrow H^{\prime}$ is a $G$-morphism (respectively weak $\mathbb{Z}^{+}, G$ )-morphism) then $\phi(G \backslash H)$ is fixed elementwise by the action of $G$ on $H^{\prime}$, and it is easy to see that we thus obtain a morphism (respectively weak $\mathbb{Z}^{+}, G$ )morphism)

$$
G \backslash \phi: G \backslash H \rightarrow G \backslash H^{\prime}
$$

THEOREM 2. The mappings $H \mapsto G \backslash H, \phi \mapsto G \backslash \phi$ constitute a functor from the category of orbit-finite G-hypergroups and G-morphisms (respectively weak $\mathbb{Z}^{+}, G$ )-morphisms) to the category of trivial G-hypergroups and hypergroup morphisms (respectively weak $\mathbb{Z}^{+}$-morphisms). 
3.6.

The following is immediate from Proposition 10.

PROPOSITION 11. If $H$ is classlike with positive integral coefficients, then so is $G \backslash H$.

\subsection{ORBITS IN STAGES}

Let $H$ be a $G$-hypergroup, and let $\psi: G_{1} \rightarrow G$ be a group homomorphism. Then putting $g_{1} \cdot x=\psi\left(g_{1}\right) \cdot x \quad\left(x \in H, g_{1} \in G_{1}\right)$ makes $H$ into a $G_{1}$-hypergroup. If $\psi\left(G_{1}\right)$ is normal in $G$, then putting $g \cdot[a]=[g a] \quad(g \in G, a \in H)$ makes $G_{1} \backslash H$ into a $G$-hypergroup, on which $\psi\left(G_{1}\right)$ acts trivially. Thus $G_{1} \backslash H$ may be regarded as a $\left(G / \psi\left(G_{1}\right)\right)$-hypergroup, and we have a natural isomorphism between $G \backslash H,\left(G / G_{1}\right) \backslash\left(G_{1} \backslash H\right)$, and $G \backslash\left(G_{1} \backslash H\right)$.

\subsection{REMARKS}

(i) Let $G$ be a group, and let it act on itself by conjugation. Then $G \backslash G$ is by definition the conjugacy class hypergroup $\bar{G}$. Relation (3.5) recovers the class equation for $G$.

(ii) If $N$ is a normal subgroup of $G_{1}$ and $c: N \rightarrow G$ is the inclusion mapping, then $G \backslash c: G \backslash N \rightarrow G \backslash G$ maps $G \backslash N$ isomorphically onto a subhypergroup of $\bar{G}$, namely, to the hypergroup of all G-classes which are contained in $N$.

(iii) $G \backslash N$ and $G \backslash \bar{N}$ are isomorphic by (i) and 3.7 .

\subsection{THE DUAL OF AN ORBIT HYPERGROUP}

Let be a finite $G$-hypergroup with coefficients in a splitting field $k$, of characteristic not dividing $\# G$. Then $k\left(G \backslash H^{*}\right)$ is the subalgebra of $k H^{*}$ consisting of those linear functionals on $k H$ that are fixed under the action of $G$ given by

$$
(g . \rho)(x)=\rho\left(g^{-1} . x\right), \quad x \in k H, \rho \in k H^{*} \text {; }
$$

see Proposition 9 above. If $\rho \in k\left(G \backslash H^{*}\right)$, then $\rho$ has a restriction to $k(G \backslash H) \in k H$ which we denote $k(\rho)$. 
THEOREM 3. The mapping $k$ is a hypergroup isomorphism of $k\left(G \backslash H^{*}\right)$ onto $k(G \backslash H)^{*}$.

Proof. We first show that $\kappa$ is surjective. Let $n \in k(G \backslash H)^{*}$. Then $\eta$ is a linear functional on $k(G \backslash H) \subseteq k H$, and so has an extension $n_{*}$ to all of $k H$. Put $\rho=(\# G)^{-1} \sum_{g \in G} g \cdot n_{*}$. Then $\rho \in k H^{*}$ and is plainly fixed by $G$, so $\rho \in k\left(G \backslash H^{*}\right)$. If $x \in k(G \backslash H)$, then $x \in k H$ and $G$ fixes $x$. Hence

$$
\rho(x)=(\# G)^{-1} \sum_{g \in G} g \cdot n_{*}(x)=\eta_{*}\left((\# G)^{-1} \sum_{g \in G} g^{-1} \cdot x\right)=n_{*}(x)=n(x) .
$$

Thus $k(\rho)=n$.

Now we show $k$ is injective. Suppose $\rho \in k\left(G \backslash H^{*}\right)$ and $\kappa(\rho)=0$; that is, that $\rho(x)=0$ whenever $x \in k H$ is fixed by $G$. Let $x \in k H$. Then

$$
\rho(y)=\left((\# G)^{-1} \sum_{g \in G} g \cdot \rho\right)(y)=\rho\left((\# G)^{-1} \sum_{g \in G} g^{-1} \cdot y\right)=0,
$$

since the argument is fixed by $G$. Hence $\rho=0$.

As an algebra, $k\left(G \backslash H^{*}\right)$ is a subalgebra of $k H^{*}$. Accordingly, if $\rho_{1}, \rho_{2} \in k\left(G \backslash H^{*}\right)$ and $x_{0} \in k H$ is group like, then $\left(\rho_{1} \rho_{2}\right)\left(x_{0}\right)=\rho_{1}(x) \rho_{2}\left(x_{0}\right)$. Now a typical grouplike in $k(G \backslash H)$ has the form $x=(\# G)^{-1} \sum_{g \in G} g \cdot x_{0}$, where $x_{0}$ is grouplike in $k H$. Thus we easily see that

$\kappa\left(\rho_{1}\right) \kappa\left(\rho_{2}\right)(x)=\kappa\left(\rho_{1}\right)(x) \kappa\left(\rho_{2}\right)(x)=\rho_{1}\left(x_{0}\right) \rho_{2}\left(x_{0}\right)=\left(\rho_{1} \rho_{2}\right)\left(x_{0}\right)=\kappa\left(\rho_{1} \rho_{2}\right)(x)$.

This shows that $K$ is an algebra map.

It is plain that $\kappa$ respects the conjugation on $k\left(G \backslash H^{*}\right)$. We have now but to show that $K$ is a cogebra map, that is, if $\gamma \in G \backslash H^{*}$ is grouplike, then $\kappa(\gamma)$ is grouplike in $(G \backslash H)^{*}$.

Now $\gamma \in k\left(G \backslash H^{*}\right)$ being grouplike signifies that for some grouplike $\tau \in k H^{*}, \gamma=(\# G)^{-1} \sum_{g \in G} g \cdot \tau$. Now $\kappa(\tau)$ is the restriction of $\tau$ to 
$k(G \backslash H)$, and $k(\gamma)=(\# G)^{-1} \sum_{g \in G} \kappa(g . \tau)$. The statement that $\tau \in k H^{*}$ is grouplike entails that $\tau: k H \rightarrow k$ is an algebra map. Consequently, if $x, y \in k(G \backslash H)$, since $x y \in k(G \backslash H)$ is fixed by $g$, we obtain

$$
\begin{aligned}
\kappa(\gamma)(x y) & =(\# G)^{-1} \sum_{g \in G}(g \tau)(x y) \\
& =(\# G)^{-1} \sum_{g \in G} \tau\left(g^{-1}(x, y)\right) \\
& =(\# G)^{-1} \sum_{g \in G} \tau(x) \tau(y) \\
& =\tau(x) \tau(y),
\end{aligned}
$$

while similarly $\kappa(\gamma)(x)=\tau(x)$ and $\kappa(\gamma)(y)=\tau(y)$. Thus $k(\gamma): k(G \backslash H) \rightarrow k$ is an algebra map, so is grouplike in $k(G \backslash H)^{*}$. This completes the proof.

3.10. ORBIT HYPERGROUPS AND QUOTIENT HYPERGROUPS

Let $H$ be an orbit finite $G$-hypergroup with positive integral coefficients. Let $J \leq H$ be a normal $G$-invariant subhypergroup of $H$. Then plainly the action of $G$ respects cosets, and hence the double ideal $V$ with which $J$ is associated is $G$-invariant. Therefore $H / J$ becomes a $G$-hypergroup under the quotient action of $G$ on $Q H / V$, and the quotient $\operatorname{map} \pi: \mathbb{Q} H \rightarrow \mathbb{Q} H / V$ is a G-morphism. That is to say $\pi: H \rightarrow H / J$ is a $G$-morphism. The following proposition is now easily proved.

PROPOSITION 12. G\J is a normal subhypergroup of $G \backslash H$, and the hypergroups

$$
G \backslash(H / J) \text { and }(G \backslash H) /(G \backslash J)
$$

are isomorphic.

The following related result is a consequence of Clifford's Theorem on restriction of characters to a normal subgroup, and is an analogue of [7, Theorem 2.7].

PROPOSITION 13. Let $G$ be a finite group, $N$ a normal subgroup of $G, \hat{G}$ the character hypergroup of $G, \hat{N}$ the character group of $N$. Let $G$ act on $N$, hence on $\hat{N}$, by conjugation. Let $J$ be the set of characters $x \in \hat{G}$ which are constant on cosets of $N$. Then $J$ is a normal subhypergroup of $\hat{G}$. 
Let $\operatorname{res}(\hat{G}, N)$ be the set of restrictions to $N$ of characters of $G$, with pointwise multiplication. Then $\operatorname{res}(\hat{G}, N)$ is a hypergroup, and indeed the three hypergroups

$$
\operatorname{res}(\hat{G}, N), G \backslash \hat{N}, \hat{G} / J
$$

are isomorphic.

\section{References}

[1] Richard Braver, "On pseudo groups", J. Math. Soc. Japan 20 (1968), 13-22.

[2] J.R. McMullen and J.F. Price, "Reversible hypergroups", Rend. Sem. Mat. Fis. Milono 47 (1977), 67-85.

[3] J.R. MCMullen and J.F. Price, "Duality for finite abelian hypergroups over splitting fields", Bulz. Austral. Math. Soc. 20 (1979), $57-70$.

[4] H. Pahlings, "Characterization of groups by their character tables I", Comm. Algebra 4 (1976), 111-153.

[5] H. Pahlings, "Characterization of groups by their character tables II", Comm. Algebra 4 (1976), 155-178.

[6] Kenneth A. Ross, "Hypergroups and centers of measure algebras", Analisi armonica e spazi di funzioni su gruppi localmente compatti, 189-203 (Symposia Mathematica, 22. Academic Press, London and New York, 1977).

[7] Richard L. Roth, "Character and conjugacy class hypergroups of a finite group", Arn. Math. Pura Appl. 105 (1975), 295-311.

[8] René Spector, "Apercu de la théorie des hypergroupes", Analyse harmonique sur les groupes de Lie, 643-673 (Séminaire NancyStrasbourg 1973-75. Lecture Notes in Mathematics, 497. Springer-Verlag, Berlin, Heidelberg, New York, 1975).

[9] Moss E. Sweedler, Hopf algebras (Benjamin, New York, 1969).

Department of Pure Mathematics, University of Sydney, Sydney, New South Wales. 